\title{
Combined Lycium barbarum polysaccharides and C-phycocyanin increase gastric Bifidobacterium relative abundance and protect against gastric ulcer caused by aspirin in rats
}

Shu-Yu Hsieh ${ }^{1}$, Yu Zhi Lian ${ }^{1}$, I-Hsuan Lin², Yu-Chen Yang ${ }^{3}$, Alexey A. Tinkov ${ }^{4,5}$, Anatoly V. Skalny 4,5 and Jane C.-J. Chao ${ }^{1,6,7^{*}}$ (D)

\begin{abstract}
Background: Non-steroidal anti-inflammatory drugs such as aspirin are used for the treatment of cardiovascular disease. Chronic use of low-dose aspirin is associated with the occurrence of gastric ulcer. The aim of this study was to investigate the healing potential of Lycium barbarum polysaccharides (LBP) from Chinese Goji berry and C-phycocyanin (CPC) from Spirulina platensis on gastric ulcer in rats.

Methods: Male Sprague-Dawley rats were divided into five groups: normal, aspirin (700 mg/kg bw), LBP (aspirin + $100 \mathrm{mg} / \mathrm{kg}$ bw/d LBP), CPC (aspirin + $50 \mathrm{mg} / \mathrm{kg}$ bw/d CPC), and MIX (aspirin + $50 \mathrm{mg} / \mathrm{kg} \mathrm{bw} / \mathrm{d}$ LBP + $25 \mathrm{mg} /$ $\mathrm{kg}$ bw/d CPC) groups. Gastric ulcer was developed by daily oral feeding of aspirin for 8 weeks. Treatments were given orally a week before ulcer induction for 9 weeks.

Results: The MIX group elevated gastric cyclooxygenase-1, prostaglandin $\mathrm{E}_{2}$, and total nitrite and nitrate levels by $139 \%, 86 \%$, and $66 \%$, respectively, compared with the aspirin group $(p<0.05)$. Moreover, the MIX group reduced lipid peroxides malondialdehyde levels by $78 \%(p<0.05)$. The treatment of LBP and/or CPC increased gastric Bifidobacterium relative abundance by $2.5-4.0$ times compared with the aspirin group $(p<0.05)$.

Conclusions: We conclude that combined LBP and CPC enhance gastroprotective factors, inhibit lipid peroxidation, and increase gastric Bifidobacterium relative abundance. Combined LBP and CPC have protective potential against gastric ulcer caused by aspirin in rats.
\end{abstract}

Keywords: Aspirin, C-phycocyanin, Gastric ulcer, Lycium barbarum polysaccharides, Microbiota

\section{Introduction}

Peptic ulcer is a common disease of the digestive system [1]. Peptic ulcer is characterized by the erosion of mucosa, submucosa, and muscular layers on the lower esophagus, stomach, or duodenum [1]. A systematic

${ }^{*}$ Correspondence: chenjui@tmu.edu.tw

${ }^{7}$ Nutrition Research Center, Taipei Medical University Hospital, Taipei 110, Taiwan

Full list of author information is available at the end of the article study of 31 articles published between January 1980 and February 2009 showed that the pooled incidence rate of uncomplicated peptic ulcer was 0.9 per 1000 person-years in the general population [2]. The lifetime prevalence of peptic ulcer diagnosed by physicians in the western countries was approximately $5-10 \%$ in the late twentieth century $[3,4]$. The prevalence of peptic ulcer assessed by upper gastrointestinal endoscopy in Taiwan from January to August 2008 was 9.4\% [5]. The prevalence of gastric ulcer, duodenal ulcer, as well as both original author(s) and the source, provide a link to the Creative Commons licence, and indicate if changes were made. The images or other third party material in this article are included in the article's Creative Commons licence, unless indicated otherwise in a credit line to the material. If material is not included in the article's Creative Commons licence and your intended use is not permitted by statutory regulation or exceeds the permitted use, you will need to obtain permission directly from the copyright holder. To view a copy of this licence, visit http://creativecommons.org/licenses/by/4.0/. The Creative Commons Public Domain Dedication waiver (http://creativeco mmons.org/publicdomain/zero/1.0/) applies to the data made available in this article, unless otherwise stated in a credit line to the data. 
gastric and duodenal ulcers in Taiwan in 2008 was 4.7\%, $3.9 \%$, and $0.9 \%$, respectively [5]. Gastric ulcer develops when the gastroprotective factors are overwhelmed by the damaging factors in the stomach [6]. The gastroprotective factors include antioxidants, bicarbonate, mucin, nitric oxide (NO), and prostaglandins (PGs) in the gastric mucosa. The damaging factors include oxidative stress, gastric acid, Helicobacter pylori, and non-steroidal antiinflammatory drugs (NSAIDs). Aspirin (acetylsalicylic acid, PubChem CID: 2244), one of NSAIDs, acts as a deleterious factor. Low-dose (75-325 mg/d) aspirin was used for the protection and therapy of cardiovascular disease. Chronic use of low-dose aspirin was associated with increased ulcer complications [7]. NSAIDs could selectively inhibit gastric cyclooxygenase (COX) activity and decrease gastric $\mathrm{PGE}_{2}$ formation [8]. In addition, aspirin could decrease gastric total nitrite and nitrate (NOx) which can act as a protective factor to stimulate gastric blood flow and mucin secretion $[9,10]$. On the other hand, aspirin increased oxidative stress by reducing gastric superoxide dismutase activity [11].

Lycium barbarum known as Chinese wolfberry or Tibetan goji berry has been used as herbal food for health promotion and traditional Chinese medicine [12]. The water-soluble active components of the extract from the fruit of Lycium barbarum L. such as polysaccharides (LBP) have been reported to have antioxidant, antiinflammatory, and immunomodulatory activity [13-15]. C-phycocyanin (CPC, PubChem SID: 53837743), a major biliprotein pigment of Spirulina platensis, was widely used in food coloring and cosmetics as the non-toxic natural dye and has been approved by Food and Drug Administration as a dietary supplement in many countries [16]. C-phycocyanin has been found to possess hepatoprotective, antioxidant, anti-inflammatory, and wound healing properties [16-18]. Due to the characteristics of free oxygen radical scavenger, anti-inflammatory, immunomodulatory, regenerative, and wound-healing activities for LBP and CPC, it is reasonably hypothesized that LBP and/or CPC may exert the recovery of gastric ulcer through lowering oxidative damage, inhibiting proinflammatory responses, improving gastroprotection, and altering gastric microbiota [13-20]. The aim of this study was to investigate whether Lycium barbarum polysaccharides and/or C-phycocyanin could have healing potential on gastric ulcer caused by aspirin in rats.

\section{Materials and methods}

\section{Animals and treatments}

Male Sprague-Dawley rats (200-250 g) aged 7 weeks old were purchased from BioLASCO Taiwan Co., Ltd. (Taipei, Taiwan), and housed in individual cages at $22-24{ }^{\circ} \mathrm{C}$ on a 12 -h light-dark cycle. After 1-week adaptation period, rats were randomly divided into five groups ( $n=10-12$ per group): normal (N) group $(n=11)$ fed with a standard powder diet, aspirin (ASP) group $(n=10)$ fed with aspirin $(700 \mathrm{mg} / \mathrm{kg} \mathrm{bw})$, LBP group $(n=12)$ fed with aspirin $(700 \mathrm{mg} / \mathrm{kg} \mathrm{bw})$ and treated with LBP $(100 \mathrm{mg} / \mathrm{kg}$ bw/d dissolved in $1 \mathrm{~mL}$ water) [21], CPC group $(n=12)$ fed with aspirin $(700 \mathrm{mg} / \mathrm{kg}$ bw) and treated with CPC $(50 \mathrm{mg} / \mathrm{kg}$ bw/d dissolved in $1 \mathrm{~mL}$ water) [22, 23], and MIX group $(n=11)$ fed with aspirin $(700 \mathrm{mg} / \mathrm{kg} \mathrm{bw})$ and treated with LBP $(50 \mathrm{mg} /$ $\mathrm{kg} \mathrm{bw} / \mathrm{d})+$ CPC $(25 \mathrm{mg} / \mathrm{kg} \mathrm{bw} / \mathrm{d})$. The dosage of LBP at $100 \mathrm{mg} / \mathrm{kg} \mathrm{bw} / \mathrm{d}$ showed antioxidant effects [21]. The dosage of CPC at $50 \mathrm{mg} / \mathrm{kg} \mathrm{bw/d} \mathrm{had} \mathrm{antioxidant} \mathrm{and}$ anti-inflammatory effects $[22,23]$. The normal group was fed with a standard powder diet (Laboratory Rodent 5001 powder diet, PMI Nutrition International Inc., Brentwood, MO, USA) containing $48.7 \%(\mathrm{w} / \mathrm{w})$ carbohydrate (58.0\% total calorie), $23.9 \%(\mathrm{w} / \mathrm{w})$ protein $(28.5 \%$ total calorie), and $5.0 \%(\mathrm{w} / \mathrm{w})$ fat (13.5\% total calorie) for 9 weeks. The aspirin group was fed with aspirin (A5376, SigmaAldrich, St. Louis, MO, USA) which was mixed into the feed after 12-h fasting from week 2 to week 9 to develop gastric ulcer. The aspirin group and the treatment groups were given by daily gavage feeding of water or treatment, respectively, in a total volume of $1 \mathrm{~mL}$ at 9:00 am from week 1 to week 9 , and aspirin was given by mixing into the feed 30-min after gavage feeding from week 2 to week 9. Lycium barbarum polysaccharides extract containing $40 \%$ polysaccharides were purchased from the commercial source (GojiMax ${ }^{\circledR} 40 \%$, GOJ-01-040POLYS, Priority Healthfood Cor., New Taipei, Taiwan), and obtained by water extraction. The total polysaccharides were determined spectrophotometrically.

The powder of LBP extract was suspended in deionized water, and the suspension was mixed with $8 \mathrm{M}$ trifluoroacetic acid $(1: 1)$ at $110{ }^{\circ} \mathrm{C}$ for $4 \mathrm{~h}$ to hydrolyze polysaccharides into monosaccharides. The compositions and concentrations $(\mu \mathrm{g} / \mathrm{mg})$ of monosaccharides were determined using high $\mathrm{pH}$ anion exchange chromatographypulsed amperometric detection (HPAEC-PAD) [24], and different monosaccharides were used as standards. The amount $(\mu \mathrm{g} / \mathrm{mg})$ of total monosaccharides before and after hydrolysis was measured by Academia Sinica (Taipei, Taiwan) using HPAEC-PAD, and total carbohydrate was determined by the difference in monosaccharides between after and before hydrolysis. The compositions of monosaccharides were expressed as the percentage of total carbohydrate, and mentioned previously as $92.04 \%$ glucose, $4.84 \%$ mannose, $1.82 \%$ galactose, $0.89 \%$ arabinose, and $0.41 \%$ rhamnose [24].

C-phycocyanin was generously supplied by Far East Bio-Tec. Co., Ltd. (Taipei, Taiwan), and contained 25\% C-phycocyanin. The powder of Spirulina platensis was 
mixed with sterile water for $24 \mathrm{~h}$, centrifuged to collect the supernatant, and dried by a freeze dryer. Water was sterilized at $121{ }^{\circ} \mathrm{C}$ for $30 \mathrm{~min}$. The powder of C-phycocyanin contained $40 \%$ protein $(62 \% \mathrm{C}$-phycocyanin and $38 \%$ other unidentified proteins), $25 \%$ polysaccharides, $6 \%$ nucleotides, $5 \%$ ash, $5 \%$ water, and $19 \%$ other unidentified ingredients. Feed intake was recorded daily, and body weight was measured weekly. After 9-week experimental period, rats were anesthetized by intraperitoneal injection with Zoletil $50(1 \mathrm{~mL} / \mathrm{kg} \mathrm{bw})$ and Rompun $(0.5 \mathrm{~mL} / \mathrm{kg} \mathrm{bw})$ after 12 -h fasting and then sacrificed. Blood samples ( $n=10-12$ per group) were collected from the tail vein, allowed to be clotted at room temperature, and centrifuged at $1000 \mathrm{~g}$ at $4{ }^{\circ} \mathrm{C}$ for $10 \mathrm{~min}$. Serum was separated in the supernatant and used for hydroxyproline analysis. The whole stomach tissue ( $n=10-12$ per group) was excised for ulcer assessment and biochemical analyses. The excised stomach tissue $(5 \mathrm{~mm} \times 5 \mathrm{~mm})$ from the normal or lesion area was preserved in $10 \%$ buffered formalin for histopathological analysis. The remaining stomach tissue was aliquoted and stored at $-20{ }^{\circ} \mathrm{C}$ for further biochemical analysis. Gastric mucosa $(n=5$ per group) was collected by scraping using a glass slide and used for total genomic DNA extraction in gastric microbiota analysis. All animal protocols were approved by the Institutional Animal Care and Use Committee at Taipei Medical University (LAC-2015-0214).

\section{Ulcer assessment}

The formation of gastric ulcer was assessed pathologically using both ulcer index (UI) and ulcer score. The whole stomach tissue was harvested, opened along the greater curvature, and the mucosa was exposed for ulcer evaluation. The opened stomach tissue was fixed on the cork board with fine needles, and red coloration and hemorrhagic streaks in the ulcerated area were observed. The ulcerated area was assessed using planimetry under $1 \mathrm{~mm} \times 1 \mathrm{~mm}$ graph paper with recording camera. Ulcer index was calculated using ImageJ software as below [25]:
The development of gastric ulcer was monitored biochemically using serum hydroxyproline levels. Hydroxyproline has been considered as a serum biomarker for gastric ulcer in rats [26]. A decrease in serum hydroxyproline levels reflected the development of gastric ulcer by aspirin, alcohol, or stress in rats [26]. Serum hydroxyproline were assessed by an enzymatic colorimetric method using a commercial reagent kit (CSB-E08838r, Cusabio Biotech Co., Ltd., College Park, MD, USA), and the absorbance was measured at $450 \mathrm{~nm}$ and corrected at $540 \mathrm{~nm}$.

\section{Gastroprotective factors in stomach tissue}

Cyclooxygenase-1 (COX-1), cyclooxygenase-2 (COX2 ), prostaglandin $\mathrm{E}_{2}\left(\mathrm{PGE}_{2}\right)$, and total nitrite and nitrate (NOx) levels as gastroprotective factors in stomach tissue were determined. The stomach tissue $(100 \mathrm{mg})$ was homogenized in phosphate buffered saline (PBS) $\left(1 \mathrm{~mL}\right.$ ), centrifuged at $5000 \mathrm{~g}$ at $4{ }^{\circ} \mathrm{C}$ for $5 \mathrm{~min}$, and the supernatant was collected for COX-1, COX-2, and PGE 2 analyses. All analyses were measured by enzymatic colorimetric methods using commercial reagent kits (CSBE1346r for COX-1, E3399r for COX-2, and E07967r for $\mathrm{PGE}_{2}$, Cusabio Biotech Co., Ltd., College Park, MD, USA), and the absorbance was analyzed at $450 \mathrm{~nm}$ and corrected at $540 \mathrm{~nm}$ for COX-1 [27] and COX-2 [28] and at $600 \mathrm{~nm}$ for $\mathrm{PGE}_{2}$ [29]. The stomach homogenate was centrifuge at $10,000 \mathrm{~g}$ at $4{ }^{\circ} \mathrm{C}$ for $20 \mathrm{~min}$, the supernatant was subsequently centrifuged at $100,000 \mathrm{~g}$ for $30 \mathrm{~min}$ at $4{ }^{\circ} \mathrm{C}$, and the supernatant was finally used for total nitrite and nitrate analysis. Gastric total nitrite and nitrate levels which represent the final metabolites of nitric oxide oxidation were assessed by an enzymatic colorimetric method using a commercial kit with Griess reagent (No 780001, Cayman Chemical, Ann Arbor, MI, USA), and the absorbance was measured at $540 \mathrm{~nm}$ [29]. All measurements are expressed as weight or concentration per $\mathrm{mg}$ protein in stomach tissue. Protein content in stomach tissue was determined by Bradford assay [30] using a commercial reagent kit (No 500-0113, Bio-Rad Laborato-

Ulcer index $=10 / x$, where $x=$ total mucosal area/total ulcerated area.

The excised stomach tissue $(5 \mathrm{~mm} \times 5 \mathrm{~mm})$ from the normal or lesion area was preserved in $10 \%$ buffered formalin overnight, embedded in paraffin wax, and stained with H\&E to observe the damaged stomach. Coded specimens were evaluated by a pathologist in a blinded fashion. Ulcer score was evaluated under a light microscope according to the following criteria: 0: normal coloration, 0.5: red coloration, 1: spot ulcers, 1.5: hemorrhagic streaks, 2: ulcers $>3 \mathrm{~mm}$ but $<5 \mathrm{~mm}$, and 3: perforation [25]. ries, Inc., Hercules, CA, USA).

\section{Gastric antioxidant markers}

The activity of superoxide dismutase (SOD) and the levels of lipid peroxides malondialdehyde (MDA) in stomach tissue were analyzed to determine antioxidant activity in different groups. The stomach tissue $(100 \mathrm{mg})$ were homogenized with the buffer $(1 \mathrm{~mL})$ containing $20 \mathrm{mM}$ 4-(2-hydroxyethyl)-1-piperazineethanesulfonic acid (HEPES), $1 \mathrm{mM}$ ethylene glycol tetraacetic acid (EGTA), 
$210 \mathrm{mM}$ mannitol, and $70 \mathrm{mM}$ sucrose, centrifuged at $1500 \mathrm{~g}$ for at $4{ }^{\circ} \mathrm{C}$ for $5 \mathrm{~min}$, and the supernatant was used for SOD analysis. The activity of SOD was measured by an enzymatic colorimetric method using a commercial reagent kit (No 706002, Cayman Chemical, Ann Arbor, MI, USA) [31]. The stomach homogenate $(10 \mu \mathrm{L})$ was mixed with xanthine oxidase $(20 \mu \mathrm{L})$ for $30 \mathrm{~min}$, and the absorbance was recorded at $450 \mathrm{~nm}$.

The stomach tissue $(100 \mathrm{mg})$ was homogenized with radioimmunoprecipitation assay buffer $(1 \mathrm{~mL})$, centrifuged at $1600 \mathrm{~g}$ at $4{ }^{\circ} \mathrm{C}$ for $10 \mathrm{~min}$, and the supernatant was analyzed for MDA levels. The levels of MDA were determined by an enzymatic colorimetric method [32] using a commercial reagent kit (No. 10009055, Cayman Chemical, Ann Arbor, MI, USA). The stomach homogenate $(10 \mu \mathrm{L})$ was added with sodium dodecyl sulfate $(10 \mu \mathrm{L})$ and color reagent $(400 \mu \mathrm{L})$ containing 2 -thiobarbituric acid and acetic acid, and incubated at $90-100{ }^{\circ} \mathrm{C}$ for $60 \mathrm{~min}$. The samples were put in ice for $10 \mathrm{~min}$ to stop the reaction, centrifuged at $1600 \mathrm{~g}$ at $4{ }^{\circ} \mathrm{C}$ for $10 \mathrm{~min}$, put at room temperature for $30 \mathrm{~min}$, and the absorbance was measured at $540 \mathrm{~nm}$.

\section{Protein expression of inflammatory markers in stomach tissue}

The inflammatory markers such as nuclear factor (NF)-kB (P65), intercellular adhesion molecule (ICAM)1 , tumor necrosis factor (TNF)- $\alpha$, interleukin (IL)-1 $\beta$, and IL-10 in stomach tissue were measured by enzymelinked immunosorbent assays. The stomach tissue (100 mg) was homogenized in PBS $(1 \mathrm{~mL})$, mixed with complete hypotonic buffer $(50 \mu \mathrm{L})$ and nonyl phenoxypolyethoxylethanol (NP-40) $(5 \mu \mathrm{L})$, and centrifuged at $14,000 \mathrm{~g}$ at $4{ }^{\circ} \mathrm{C}$ for $30 \mathrm{~s}$. The supernatant was mixed with complete nuclear extraction buffer $(10 \mu \mathrm{L})$ on ice for $30 \mathrm{~min}$, centrifuged at $14,000 \mathrm{~g}$ at $4{ }^{\circ} \mathrm{C}$ for $10 \mathrm{~min}$, and the supernatant as analyzed for NF- $\mathrm{kB}$ (P65) binding to DNA using a commercial reagent kit (No 10007889, Cayman Chemical, Ann Arbor, MI, USA) [33]. The samples were added complete transcription factor binding assay buffer at $4{ }^{\circ} \mathrm{C}$ overnight, washed in wash buffer for several times, incubated with NF-kB (P65) primary antibody for $1 \mathrm{~h}$, and then added goat anti-rabbit IgG horseradish peroxidase (HRP) conjugate secondary antibody for $1 \mathrm{~h}$ with several washes in between. Finally, the samples were added transcription factor developing solution in dark for 30 min and mixed with stop solution $\left(\mathrm{H}_{2} \mathrm{SO}_{4}\right)$, and the absorbance was recorded at $450 \mathrm{~nm}$.

The levels of ICAM-1 in stomach tissue were determined using a commercial reagent kit (CSB-E04576r, Cusabio Biotech Co., Ltd., College Park, MD, USA) [34]. The stomach homogenate $(100 \mu \mathrm{L})$ in PBS was added to a 96-well plate with pre-coated primary antibody at
$37{ }^{\circ} \mathrm{C}$ for $2 \mathrm{~h}$, incubated with biotin-conjugated antibody $(100 \mu \mathrm{L})$ specific to ICAM- 1 at $37^{\circ} \mathrm{C}$ for $1 \mathrm{~h}$, and then added HRP-avidin $(100 \mu \mathrm{L})$ at $37{ }^{\circ} \mathrm{C}$ for $1 \mathrm{~h}$ after several washes. The samples were added substrate solution $\left(3,3^{\prime}, 5,5^{\prime}\right.$-tetramethylbenzidine, TMB) in dark at $37^{\circ} \mathrm{C}$ for $15 \mathrm{~min}$, and then added stop solution (acetic acid). The absorbance was measured at $450 \mathrm{~nm}$ and corrected at $540 \mathrm{~nm}$.

The levels of TNF- $\alpha$, IL-1 $\beta$, and IL-10 in stomach tissue were assessed using commercial reagent kits (DY510 for TNF- $\alpha$, DY501 for IL-1 $\beta$, DY522 for IL-10, R\&D Systems, Inc., Minneapolis, MN, USA) [35]. The stomach homogenate $(100 \mu \mathrm{L})$ in PBS was added to a 96-well plate with pre-coated primary antibody for $2 \mathrm{~h}$, mixed with detection antibody $(100 \mu \mathrm{L})$ for $2 \mathrm{~h}$, and then incubated with streptavidin-HRP $(100 \mu \mathrm{L})$ in dark for 20 min with several washes in between. The samples were added TMB substrate solution $(100 \mu \mathrm{L})$ for $20 \mathrm{~min}$, and then added stop solution $\left(50 \mu \mathrm{L} 2 \mathrm{~N} \mathrm{H}_{2} \mathrm{SO}_{4}\right)$. The absorbance was determined at $450 \mathrm{~nm}$ and corrected at $540 \mathrm{~nm}$.

\section{Taxonomic compositions and diversity of gastric microbiota}

The gastric microbiota composition before and after treatments ( $n=5$ per group) was profiled using nextgeneration sequencing (NGS) approach. Total genomic DNA in gastric mucosa was extracted and purified by a commercial kit (No 51704, QIAGEN GmbH, Hilden, Germany). The $16 \mathrm{~S}$ rRNA gene amplicons were generated by domain-level polymerase chain reaction (PCR) from genomic DNA for the Illumina MiSeq System. The V3-V4 region of the bacterial 16S rRNA genes was amplified by PCR using $16 \mathrm{~S}$ rRNA primers (forward primer 341F: 5'-CCTACGGGNGGCWGCAG-3', reverse primer 805R: 5'-GACTACHVGGGTATCTA ATCC $\left.-3^{\prime}\right)$. The PCR was performed by denaturation at $95{ }^{\circ} \mathrm{C}$ for $30 \mathrm{~s}$, annealing at $55^{\circ} \mathrm{C}$ for $30 \mathrm{~s}$, and elongation at $72{ }^{\circ} \mathrm{C}$ for $30 \mathrm{~s}$ for 25 cycles. The amplified product was ligated into a sequencing primer, and a sequencing library was constructed followed by NGS sub-generation sequencing genotype analysis using an Illumina MiSeq sequencer. The microbial strains were identified using the SILVA database (v128) [36], and multiple sequence alignment analysis of microbial strains was performed by Decipher package (v2.2.0). The phylogenetic tree was constructed from the alignment using phangorn package (v2.2.0) [37]. The taxonomy assignment and phylogenetic tree were consolidated into phyloseq package, and community analyses were conducted using phyloseq package (v1.19.1) [38]. The sequence fragment reads were classified, and the resulting counts of classified reads were used to estimate relative abundance. 
Diversity of gastric microbiota was determined by alpha and beta diversity. The alpha diversity represents the differences within a microbial ecosystem to estimate how many different species could be detected in a microbial ecosystem (species richness) and how different the microbes are distributed in a microbial ecosystem (species diversity). Species richness was estimated by observed and Chaol indices, and species diversity was determined by Shannon and Simpson's indices using phyloseq package. The beta diversity indicates the differences between microbial communities from different environments to estimate how different the microbial composition is in one environment compared with another. The UniFrac distance is a distance metric for comparing biological communities, and was calculated using GUniFrac package (v1.1) to evaluate the community dissimilarity between different groups [39]. Principal coordinate analysis (PCoA) ordination on unweighted and weighted UniFrac distance was conducted using the adonis and betadisper functions of the vegan package (v2.4, https:// CRAN.R-project.org/package=vegan) to analyze the dissimilarity of composition among different groups and the homogeneity of dispersion, respectively. Comparisons of microbiota enrichment were analyzed to screen bacterial species which are most likely to explain the differences among different groups using the linear discriminant analysis (LDA) effect size (LEfSe) method. The results were visualized as a cladogram using GraPhlAn [40]. The logarithmic LDA score $>2$ is considered significantly different at $p<0.05$ [41].

\section{Statistical analysis}

Data are expressed as mean \pm standard error of means (SEM). The mean differences in body weight, food intake, ulcer assessment, and biochemical parameters among different groups ( $n=10-12$ per group) were analyzed by one-way analysis of variance (ANOVA) followed by Fisher's least significant difference test. Comparisons of the differences in relative abundance, alpha diversity, and beta diversity of gastric microbiota among different groups ( $n=5$ per group) were performed by KruskalWallis one-way ANOVA followed by Wilcoxon-MannWhitney test. All statistical analyses were performed using SAS 9.4 (SAS Institute Inc., Cary, NC, USA). Statistical significance is assigned at $p<0.05$.

\section{Results}

Body weight, food intake, and aspirin intake

There were no significant differences in body weight at weeks 0 and 1 among different groups (Table 1). Body weight of rats in all aspirin-treated groups was significantly decreased by $14 \%-19 \%$ compared with that in the normal group at week $9(p<0.05)$. Body weight gains from week 0 to week 9 were reduced in all aspirin treated groups compared with those in the $\mathrm{N}$ group $(p<0.05)$. Accumulated body weight gains are shown in Additional file 1: Fig. S1. Body weight gains were decreased at week 2 in all the groups compared with those at week 1 due to aspirin feeding at week 1 . Body weight gains were gradually increased after week 2 in the $\mathrm{N}$ group, and significantly higher from week 3 to week 9 compared with those in the ASP group $(p<0.05)$. However, body weight gains had delayed to be elevated until week 3 in all aspirin treated groups. The average daily food intake of rats was 29-31 $\mathrm{g}$ and 19-22 $\mathrm{g}$ during the pre-treatment period at week 1 and the ulcer development period from week 2 to week 9 , respectively. There was no significant difference in food intake among different groups at week 1 . However, food intake in all aspirin treated groups was significantly reduced compared with that in the normal group

Table 1 Body weight, food intake, and aspirin intake of rats in different groups

\begin{tabular}{|c|c|c|c|c|c|}
\hline & $\mathrm{N}$ & ASP & LBP & CPC & MIX \\
\hline \multicolumn{6}{|l|}{ Body weight (g/d) } \\
\hline Week 0 & $229 \pm 2$ & $232 \pm 2$ & $231 \pm 2$ & $234 \pm 2$ & $233 \pm 2$ \\
\hline Week 1 & $305 \pm 2$ & $305 \pm 2$ & $307 \pm 1$ & $308 \pm 3$ & $310 \pm 3$ \\
\hline Week 9 & $427 \pm 7$ & $344 \pm 11^{*}$ & $368 \pm 7^{*}$ & $352 \pm 11^{*}$ & $351 \pm 8^{*}$ \\
\hline Weight gain (g) & $197.3 \pm 6.9$ & $111.9 \pm 11.1^{*}$ & $137.6 \pm 7.1^{*}$ & $117.4 \pm 11.1^{*}$ & $118.5 \pm 8.4^{*}$ \\
\hline \multicolumn{6}{|l|}{ Food intake (g/d) } \\
\hline Pre-treatment (week 1) & $29.4 \pm 0.3$ & $29.4 \pm 0.5$ & $30.5 \pm 0.2$ & $30.2 \pm 0.2$ & $29.9 \pm 0.2$ \\
\hline Induction (weeks 2-9) & $21.9 \pm 0.3$ & $18.5 \pm 0.5^{*}$ & $19.8 \pm 0.2^{* \#}$ & $18.9 \pm 0.4^{*}$ & $18.5 \pm 0.4^{*}$ \\
\hline Aspirin intake (mg/kg bw/d) & - & $587 \pm 16$ & $628 \pm 8^{\#}$ & $601 \pm 13$ & $590 \pm 14$ \\
\hline Completion rate (\%) & - & $84 \pm 2$ & $90 \pm 1^{\#}$ & $86 \pm 2$ & $84 \pm 2$ \\
\hline
\end{tabular}

Data are mean \pm SEM ( $n=10-12$ per group). Differences between the groups were determined by one-way ANOVA followed by Fisher's least significant difference test $\mathrm{N}$, standard powder diet; ASP, aspirin; LBP, aspirin + Lycium barbarum polysaccharides (LBP); CPC, aspirin + C-phycocyanin (CPC); MIX, aspirin + LBP + CPC ${ }^{*} p<0.05$ compared with the $\mathrm{N}$ group; ${ }^{\#} p<0.05$ compared with the ASP group 
$(p<0.05)$. Additionally, food intake in the LBP treated group was significantly increased by $7 \%$ compared with that in the ASP group $(p<0.05)$. Aspirin intake completion rate is the percentage of aspirin actual intake to the assigned dosage of aspirin. The completion rate of aspirin intake was $84 \%-90 \%$ in different groups. Corresponding to an increase in food intake by $7 \%$ in the LBP group, both aspirin intake and completion rate in the LBP group were significantly increased by $7 \%$ compared with those in the ASP group $(p<0.05)$.

\section{Effects of LBP and/or CPC on ulcer index and serum hydroxyproline}

Red coloration and hemorrhagic streaks in the gastric mucosal surface were observed for the formation of ulcer in different groups. There was no redness and swelling on the gastric surface in the $\mathrm{N}$ group, however, the hemorrhagic streaks were obvious in the ASP group, and redness was observed in the LBP, CPC, and MIX groups (Additional file 1: Fig. S2a). The histological staining of stomach tissue is shown in Additional file 1: Fig. S2b, and corresponding ulcer scores are indicated in Fig. 1b. The ASP group had significantly higher ulcer index $(p<0.001)$ and ulcer score $(p<0.01)$ than the $\mathrm{N}$ group (Fig. 1a). The LBP group had significantly lower ulcer score than the ASP group $(0.5 \pm 0.2$ vs. $1.9 \pm 0.2$, $p<0.01$ ) (Fig. 1b). There were no significant differences in ulcer index between the LBP and/or CPC treated groups and the ASP group (Fig. 1a). However, the LBP $(p=0.05)$ and MIX groups $(p=0.07)$ tended to reduce ulcer index compared with the ASP group (Fig. 1a). Serum hydroxyproline levels as a biochemical index of gastric ulcer were significantly decreased by $24 \%$ and $26 \%$ in the ASP and $\mathrm{CPC}$ groups, respectively, compared with the $\mathrm{N}$ group $(p<0.05)$ (Fig. 1c).

\section{Effects of LBP and/or CPC on gastroprotective factors in stomach tissue}

Gastric COX-1 levels were significantly elevated by 2.3 and 2.9 times in the CPC and MIX groups, respectively, compared with those in the $\mathrm{N}$ group $(p<0.05)$ (Fig. 2a). The MIX group had higher gastric COX-1 levels than the ASP group ( 3.80 vs. $1.59 \mathrm{ng} / \mathrm{mg}$ protein) $(p<0.05)$. Gastric COX-2 levels did not differ among different groups (Fig. 2b). Gastric PGE $_{2}$ levels were significantly increased by $155 \%$ or $86 \%$ in the MIX group compared with the
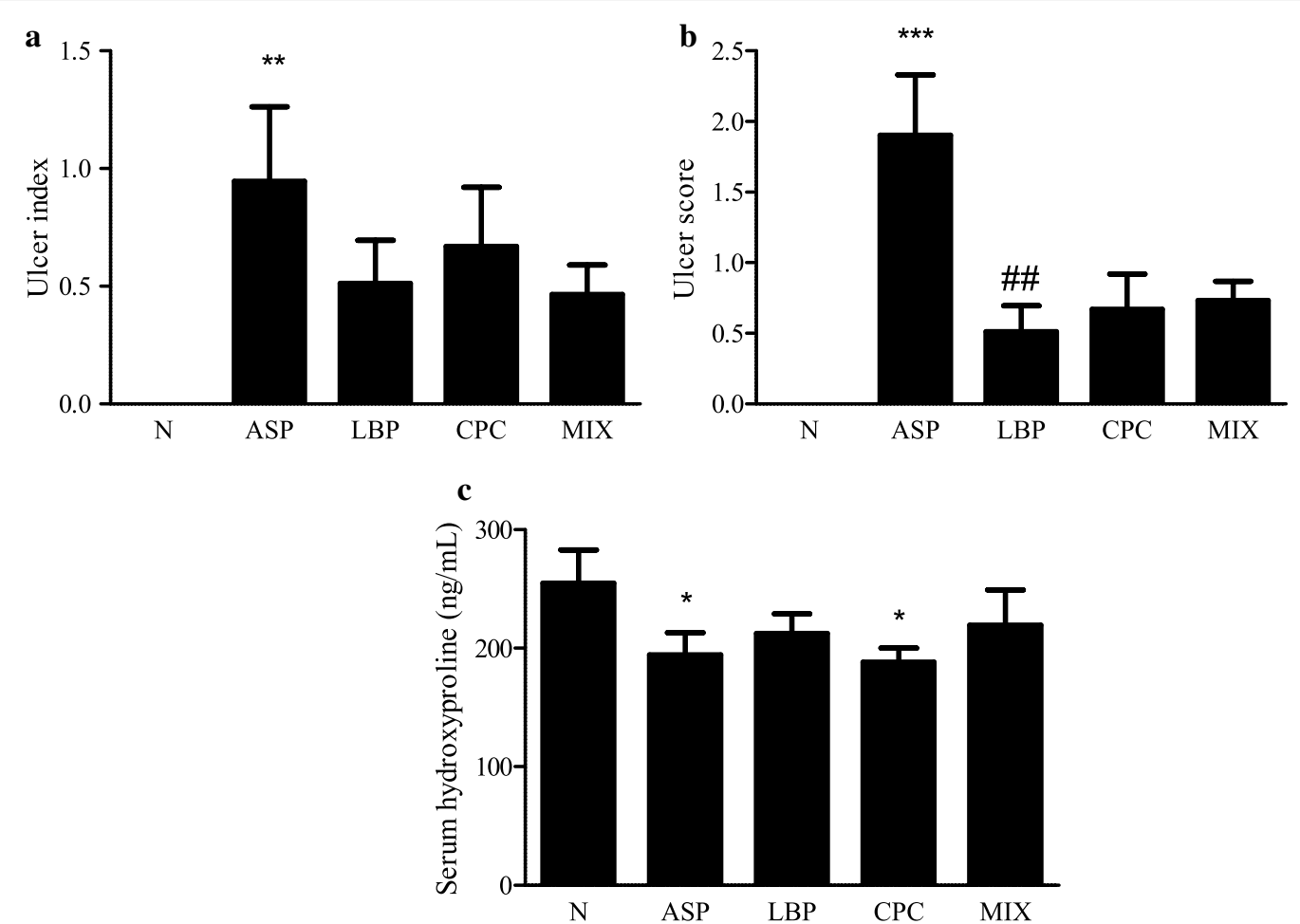

Fig. 1 Effects of Lycium barbarum polysaccharides (LBP) and/or C-phycocyanin (CPC) on a ulcer index, b ulcer score, and c serum hydroxyproline level in stomach tissue. N, standard powder diet; ASP, aspirin; LBP, aspirin + Lycium barbarum polysaccharides (LBP); CPC, aspirin + C-phycocyanin $(C P C)$; MIX, aspirin + LBP + CPC. Data are mean \pm SEM ( $n=10-12$ per group). Differences between the groups were determined by one-way ANOVA followed by Fisher's least significant difference test. ${ }^{*} p<0.05$ compared with the N group; ${ }^{* *} p<0.01$ compared with the $N$ group; ${ }^{* * *} p<0.001$

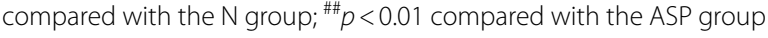




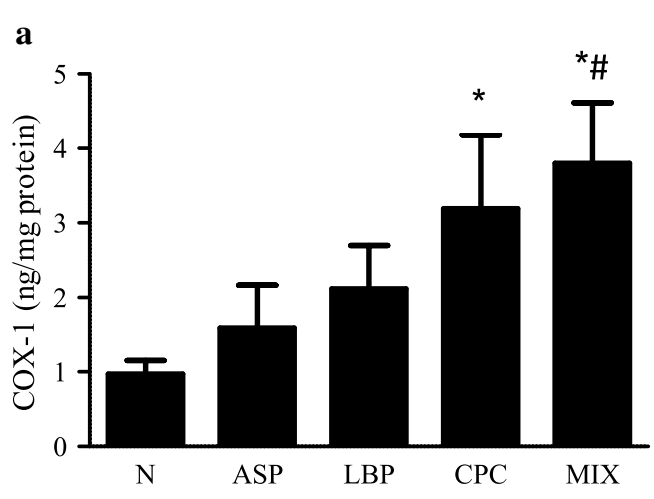

b
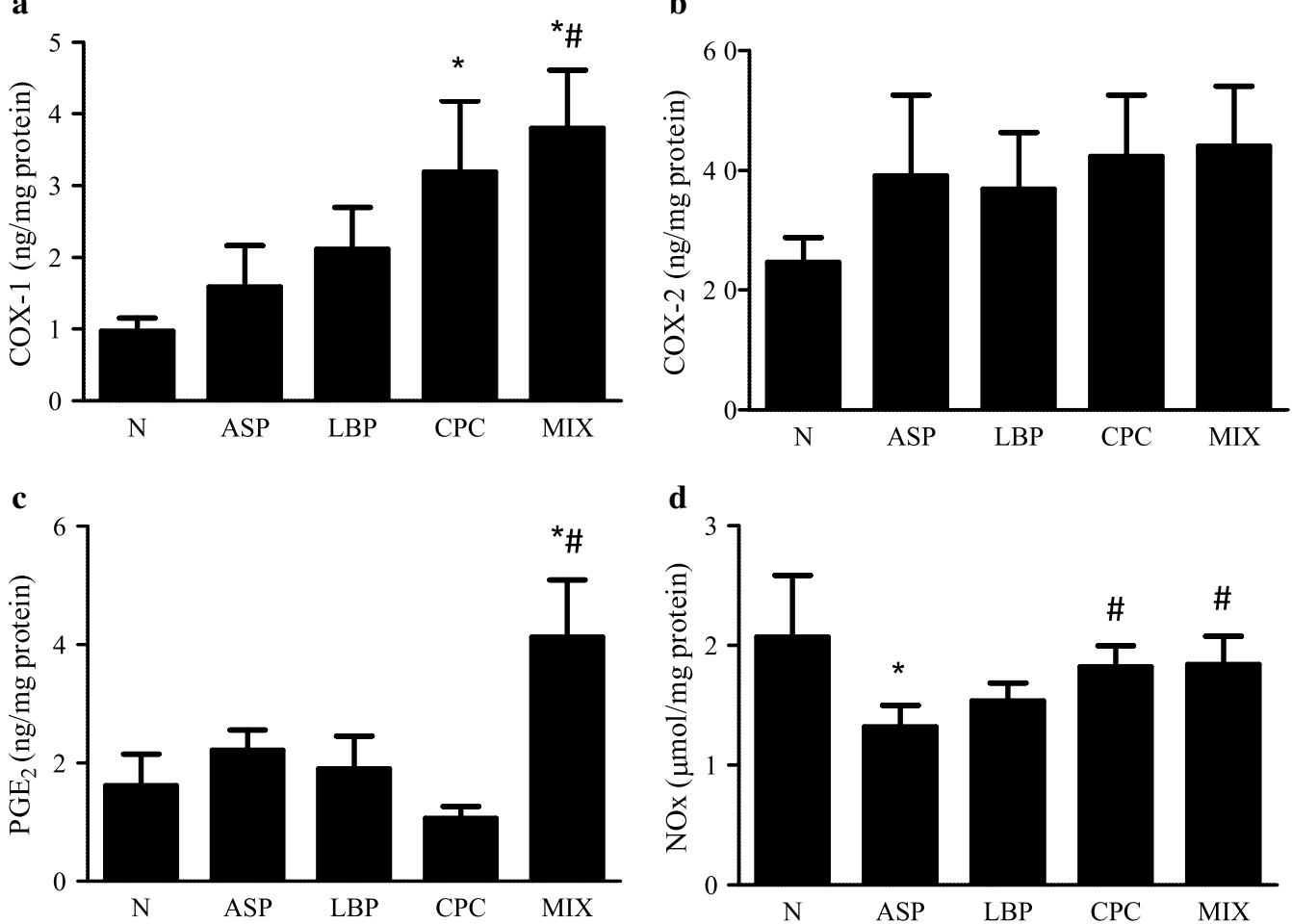

d

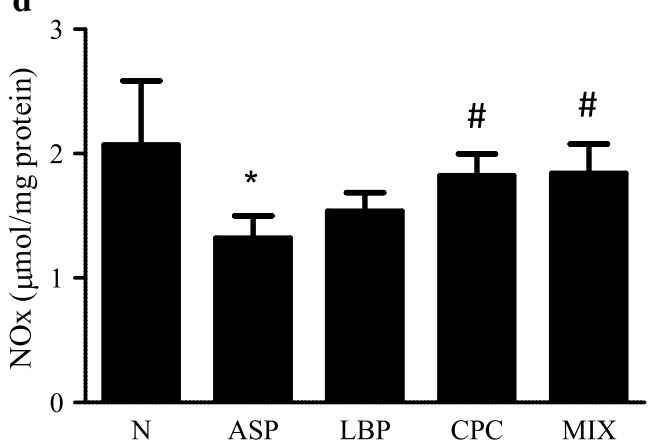

Fig. 2 Effects of Lycium barbarum polysaccharides (LBP) and/or C-phycocyanin (CPC) on the levels of a cyclooxygenase-1 (COX-1), b cyclooxygenase-2 (COX-2), $\mathbf{c}$ prostaglandin $\mathrm{E}_{2}\left(\mathrm{PGE}_{2}\right)$, and $\mathbf{d}$ total nitrite and nitrate (NOx) in stomach tissue. $\mathrm{N}$, standard powder diet; ASP, aspirin; LBP, aspirin + Lycium barbarum polysaccharides (LBP); CPC, aspirin + C-phycocyanin (CPC); MIX, aspirin + LBP + CPC. Data are mean \pm SEM ( $n=10-12$ per group). Differences between the groups were determined by one-way ANOVA followed by Fisher's least significant difference test. ${ }^{*} p<0.05$ compared with the N group; $p<0.05$ compared with the ASP group

$\mathrm{N}$ or ASP group, respectively $(p<0.05)$ (Fig. $2 \mathrm{c})$. Gastric total nitrite and nitrate levels were significantly reduced by $38 \%$ in the ASP group compared with the $\mathrm{N}$ group $(p<0.05)$ (Fig. 2d). Both CPC and MIX groups significantly increased gastric total nitrite and nitrate levels by $64 \%$ and $66 \%$, respectively, compared with the ASP group $(p<0.05)$.

\section{Effects of LBP and/or CPC on gastric antioxidant markers}

Gastric SOD activity in the ASP and LBP groups were significantly decreased by $42 \%$ and $49 \%$, respectively, compared with that in the $\mathrm{N}$ group $(p<0.05)$ (Fig. 3a). However, there were no significant differences in gastric SOD activity among the ASP treated groups. Gastric MDA levels in the ASP groups were significantly increased by 1.1 times compared with those in the $\mathrm{N}$ group $(p<0.05)$ (Fig. $3 \mathrm{~b})$. The CPC and MIX groups significantly reduced gastric MDA levels by $85 \%$ and $78 \%$, respectively, compared with the ASP group $(p<0.05)$, and the LBP group tended to decrease gastric MDA levels compared with the ASP group $(p=0.06)$.
Effects of LBP and/or CPC on gastric inflammatory markers There were no significant differences in gastric NF- $\mathrm{B}$ (p65) activity, ICAM-1, TNF- $\alpha$, IL-1 $\beta$, and IL-10 levels (Additional file 1: Fig. S3) among different groups.

\section{Effects of LBP and/or CPC on taxonomic compositions and diversity of gastric microbiota}

The taxa distribution of rat gastric microbiota by phylum, class, family, and genus is shown in Additional file 1: Fig. S4. Firmicutes, Bacteroidetes, Proteobacteria, and Actinobacteria were the major phyla in gastric microbiota in the $\mathrm{N}$ or ASP treated groups (Fig. 4a, Additional file 1: Fig. $\mathrm{S} 4 \mathrm{a})$. The relative abundance of the phylum Firmicutes in the LBP group was significantly decreased compared with the $\mathrm{N}$ group $(p<0.05)$ (Fig. $4 \mathrm{a})$. However, the relative abundance of the phylum Actinobacteria in the LBP, CPC, and MIX groups were significantly increased compared with the ASP group $(p<0.05)$. The relative abundance of the phyla Bacteroidetes and Proteobacteria was not significantly different among different groups. The relative abundance of the genus Bifidobacterium in the 

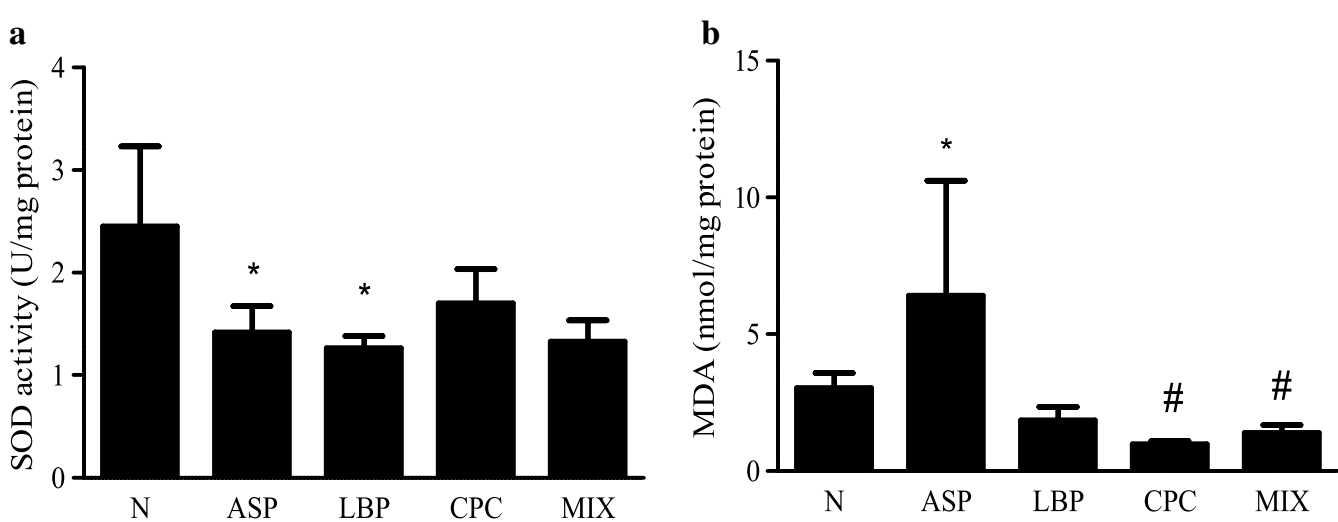

Fig. 3 Effects of Lycium barbarum polysaccharides (LBP) and/or C-phycocyanin (CPC) on gastric antioxidative markers. a Superoxide dismutase (SOD) activity and $\mathbf{b}$ malondialdehyde (MDA) levels. N, standard powder diet; ASP, aspirin; LBP, aspirin + Lycium barbarum polysaccharides (LBP); CPC, aspirin + C-phycocyanin (CPC); MIX, aspirin + LBP + CPC. Data are mean \pm SEM ( $n=10-12$ per group). Differences between the groups were determined by one-way ANOVA followed by Fisher's least significant difference test. ${ }^{*} p<0.05$ compared with the N group; ${ }^{*} p<0.05$ compared with the ASP group

LBP, CPC, and MIX groups was significantly elevated by $3.5,4.0$, and 2.5 times, respectively, compared with the ASP group $(p<0.05)$ (Fig. 4 b). There were no significant differences in the relative abundance of the genus Bifidobacterium between the $\mathrm{N}$ and ASP groups. The relative abundance of the genus Streptococcus was not significantly different between any two groups (data were not shown).

The alpha diversity was determined by species richness (observed and Chaol indices) and species diversity (Shannon and Simpson's indices). Only Simpson's indices were significantly different among different groups (Fig. 5). The Simpson's index values were significantly higher in all the ASP treated groups compared with the N group $(p<0.05)$. The beta diversity of gastric microbiota is indicated in Additional file 1: Fig. S5. The beta diversity of gastric microbiota in unweighted and weighted UniFrac distances was not significantly different among five groups using Kruskal-Wallis one-way ANOVA $(p>0.05)$.

Gastric microbiota enrichment in different groups is shown in Fig. 6 using the linear discriminant analysis (LDA) effect size (LEfSe) method. A total of 19 significant enrichment taxa with LDA score $>2$ in the treated groups were found (Fig. 6a). There were no significant differences in LDA scores in the N or ASP group compared with 3 treated groups. The LBP group had 10 significant enrichment taxa including the phylum Actinobacteria, the class Erysipelotrichia, the order Erysipelotrichales, the family Erysipelotrichaceae, and the genus Turicibacter of the phylum Firmicutes. The CPC group had 7 significant enrichment taxa including the class Actinobacteria, the order Bifidobacteriales, and the family Bifidobacteriaceae of the phylum Actinobacteria. The MIX group had 2 significant enrichment taxa including the genera Blautia and Coprococcus of the phylum Firmicutes. As compared with the ASP or treated groups (LBP, CPC, and MIX groups together), the $\mathrm{N}$ group had significant enrichment in the class Clostridia and the order Clostridiales of the phylum Firmicutes (Fig. 6b). The ASP group had significantly higher in genus Ruminococcaceae of the phylum Firmicutes compared with the $\mathrm{N}$ or treated groups. The treated groups had significantly higher in the phylum and class Actinobacteria, the order Bifidobacteriales, the family Bifidobateriaceae, and the genera Bifidobacterium, Coriobacteriaceae, and Adlercreutzia of the phylum Actinobacteria compared with the $\mathrm{N}$ or ASP group.

\section{Discussion}

Our study showed that oral administration of aspirin $(700 \mathrm{mg} / \mathrm{kg} \mathrm{bw})$ for 8 weeks led to the development of gastric ulcer. Similarly, a previous study found that serum hydroxyproline levels were significantly reduced in rats with aspirin-induced gastric ulcer by a single oral administration $(300 \mathrm{mg} / \mathrm{kg} \mathrm{bw})$ [42]. Aspirin could decrease the gastroprotective factor such as total nitrite and nitrate levels in the stomach and increase oxidative stress by decreasing gastric SOD activity and increasing gastric lipid peroxides to result in the development of gastric ulcer. Although the treated groups had the tendency to decrease gastric ulcer index and ulcer scores compared with the ASP group, only the LBP group significantly decreased gastric ulcer score. However, the LBP group did not increase serum hydroxyproline levels after 9-week treatment period. The reason for not significant difference in gastric ulcer index and ulcer scores by LBP and/or CPC treatments was speculated 


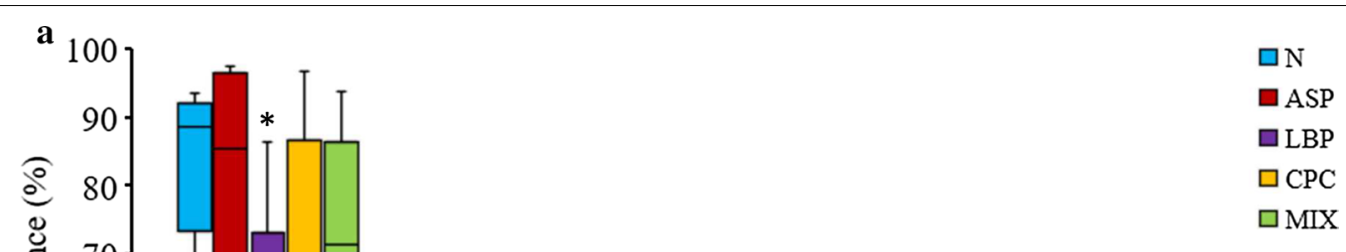

Fig. 4 Effects of Lycium barbarum polysaccharides (LBP) and/or C-phycocyanin (CPC) on relative abundance of a major phyla and $\mathbf{b}$ Bifidobacterium genus in gastric microbiota. N, standard powder diet; ASP, aspirin; LBP, aspirin + Lycium barbarum polysaccharides (LBP); CPC, aspirin + C-phycocyanin (CPC); MIX, aspirin + LBP + CPC. The horizontal lines in a boxplot from the bottom to the top represent the 25th percentile, median, and the 75th percentile of diversity values in each group ( $n=5$ per group). Relative abundance (\%) of major phyla is percentage distribution of bacteria in the classification to all microbiota. Differences between the groups were determined by Kruskal-Wallis one-way ANOVA followed by Wilcoxon-Mann-Whitney test. ${ }^{*} p<0.05$ compared with the N group; ${ }^{*} p<0.05$ compared with the ASP group

that the 9-week treatment period might be too short to observe complete healing of gastric ulcer caused by aspirin in terms of pathological observation. The clinical therapy for the patients with Helicobacter pyloriinfected peptic ulcer took 10-14 days to eradicate Helicobacter pylori [43]. For the patients with dyspepsia but no alarm symptoms such as bleeding, perforation, obstruction, and malignancy in the gastrointestinal tract, antisecretory therapy such as proton pump inhibitors and histamine $\mathrm{H}_{2}$ blockers was administered for 4-8 weeks [44]. However, up to $25 \%$ of gastric ulcer patients who continuously took NSAIDs during therapy 


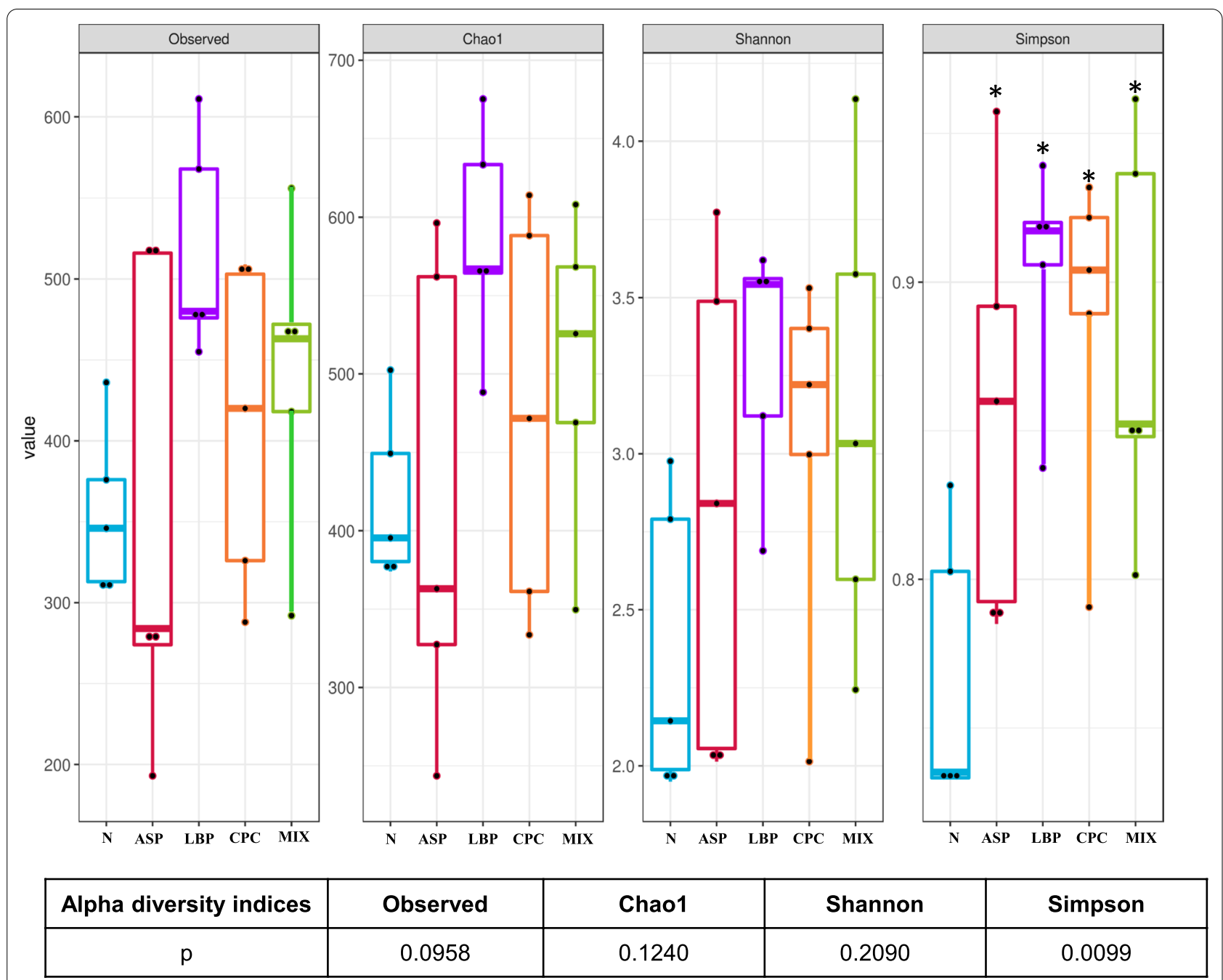

Fig. 5 Alpha diversity of gastric microbiota. Species richness was measured by observed and Chao1 indices. Species diversity was determined by Shannon and Simpson's indices. N, standard powder diet; ASP, aspirin; LBP, aspirin + Lycium barbarum polysaccharides (LBP); CPC,

aspirin + C-phycocyanin (CPC); MIX, aspirin + LBP + CPC. The horizontal lines in a boxplot from the bottom to the top represent the 25 th percentile, median, and the 75th percentile of diversity values in each group ( $n=5$ per group). The bottom and top solid dots indicate the minimum and maximum values, respectively. The $p$ value $<0.05$ is considered statistically significant using Kruskal-Wallis one-way ANOVA. ${ }^{*} p<0.05$ compared with the $\mathrm{N}$ group by Wilcoxon-Mann-Whitney test

needed more than 8 weeks for proton pump inhibitor therapy [43].

Serum hydroxyproline levels as a biomarker for gastric ulcer were significantly decreased by CPC treatment in the present study. Similarly, a previous study found that $\mathrm{CPC}$ reduced lung hydroxyproline levels as a biomarker for collagen deposition or fibrosis and had anti-fibrotic effects on bleomycin-induced pulmonary fibrosis in mice by mediating toll-like receptor 2 signaling pathway [44]. It is hypothesized that gastric mucosal healing could be affected by CPC due to its anti-fibrotic effects, and gastric ulcer score did not significantly decreased by CPC.
Our data showed that chronic oral administration of aspirin did not significantly change gastric COX-1, COX-2, and $\mathrm{PGE}_{2}$ levels, but significantly reduced gastric total nitrite and nitrate levels. Aspirin is a more potent inhibitor of COX-1 than of COX-2, and the synthesis of prostaglandins could be altered due to the changes in COX-1 and/or COX-2 activities [45]. Prostaglandins synthesized by COX-1 can protect the gastric mucosa against damage, and these synthesized by COX-2 can stimulate inflammatory reaction, suggesting that both COX-1 and COX-2 might contribute to gastric mucosal defense [45]. Gastric $\mathrm{PGE}_{2}$ levels were not significantly altered by aspirin in this study probably because of the 

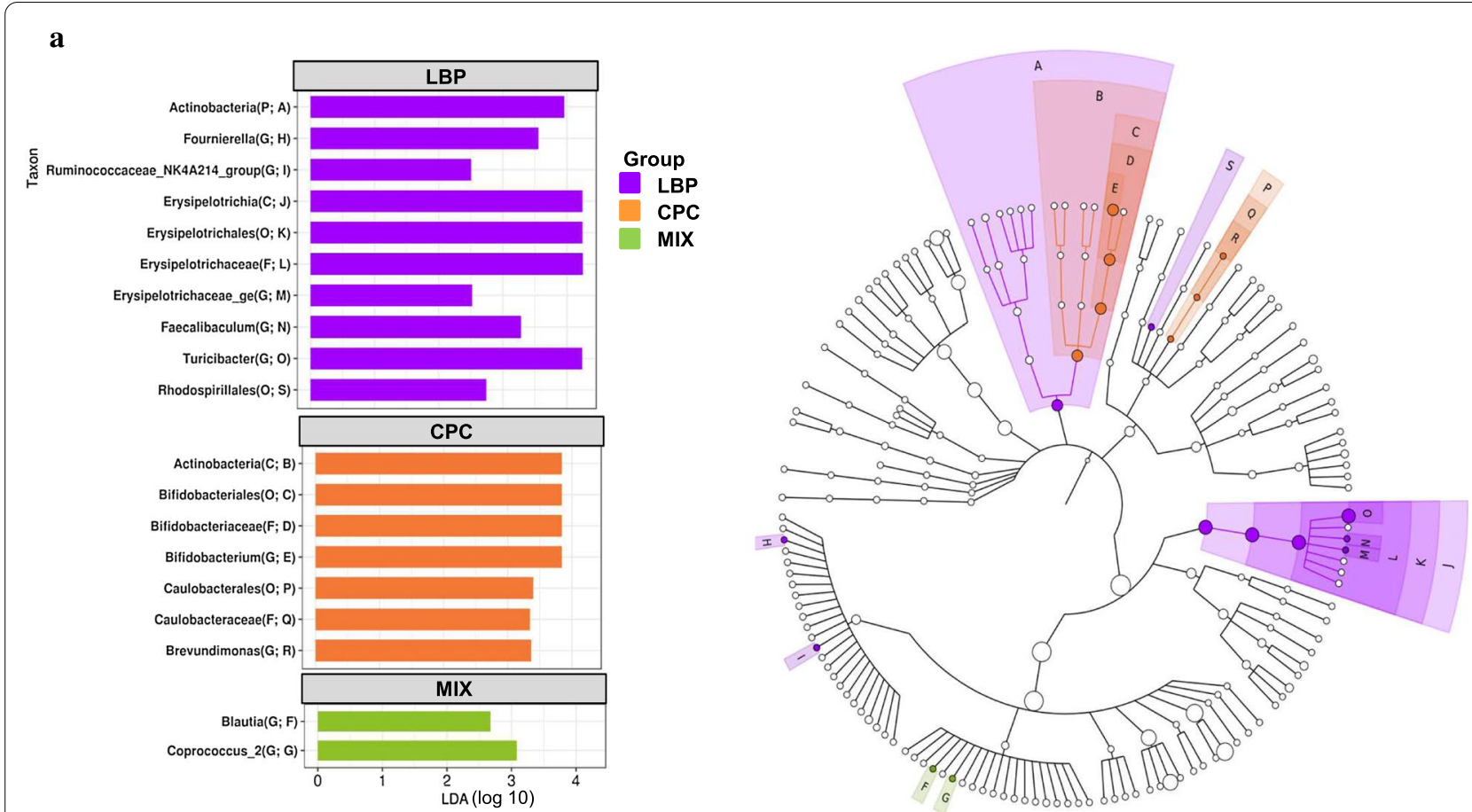

b
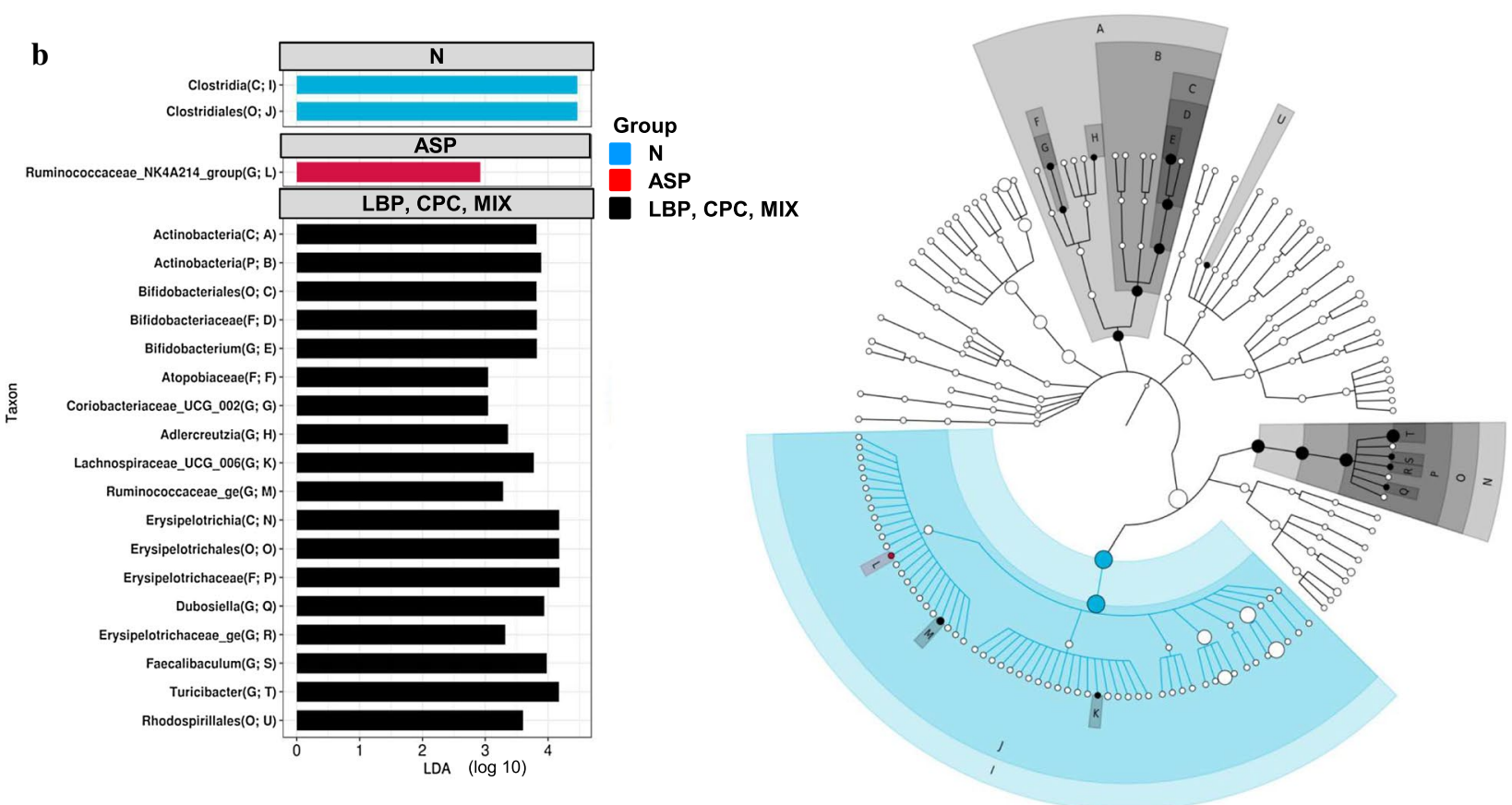

Fig. 6 Gastric microbiota enrichment in different groups ( $n=5$ per group) using the linear discriminant analysis (LDA) effect size (LEfSe). a Gastric microbiota enrichment in each group and $\mathbf{b}$ gastric microbiota enrichment in the N, ASP, and treated groups (LBP, CPC, and MIX). N, standard powder diet; ASP, aspirin; LBP, aspirin + Lycium barbarum polysaccharides (LBP); CPC, aspirin + C-phycocyanin (CPC); MIX, aspirin + LBP + CPC. C, class; $F$, family; $G$, genus; $\mathrm{O}$, order; $\mathrm{P}$, phylum. The circles of phylogenetic tree cladogram in the right panel from inside to outside represent the classification level from phylum to genus of gastric microbiota. The diameter of the circle indicates the relative abundance. The letters $A$ to $S$ in a and $A$ to $U$ in $\mathbf{b}$ represent 19 and 21 different taxa, respectively. The abbreviations in the parentheses in the left panel are taxonomy rank and position on the cladogram in the right panel, respectively. The logarithmic LDA score $>2$ shown in the left panel is considered significantly different at $p<0.05$. There were no significant differences in LDA scores in the N or ASP group compared with three treated groups in a 
change in neither COX-1 nor COX-2 levels. Consistent with our findings, a previous study revealed that gastric ulcer caused by a single oral dose $(400 \mathrm{mg} / \mathrm{kg} \mathrm{bw})$ of aspirin significantly decreased gastric total nitrite and nitrate levels, indicating gastric protection from the production of NO was reduced [10].

The MIX group significantly elevated gastric COX-1 and $\mathrm{PGE}_{2}$ levels, and the $\mathrm{CPC}$ and MIX groups also significantly increased gastric total nitrite and nitrate levels, suggesting that the combination of CPC and LBP can increase the gastroprotective factors to further protect against gastric ulcer. The previous study revealed that inhibition of NO production by NO synthase inhibitors impaired the gastroprotective effect of modafinil on the healing of gastric lesion induced by indomethacin or water-immersion stress in rats, suggesting that modulation of $\mathrm{NO}$ pathway could be potentially crucial for gastroprotection [46]. The possible mechanisms for the gastroprotective effect of $\mathrm{NO}$ against gastric ulcer could be associated with regulation of mucosal blood flow, increases in $\mathrm{COX}$ activity and $\mathrm{PGE}_{2}$ synthesis, inhibition of neutrophil infiltration, and maintenance of mucus secretion by goblet cells [46]. However, LBP did not increase gastric total nitrite and nitrate levels. It is speculated that gastric $\mathrm{pH}$ value could be affected by the production of short-chain fatty acids via the fermentation of LBP by gastric microbiota [19]. The altered gastric $\mathrm{pH}$ could weaken the potential role of LBP for the protection against gastric ulcer.

Aspirin inhibited gastric SOD activity and increased gastric lipid peroxides MDA levels. Oxidative stress is considered to be involved in the pathogenesis of gastric ulcer, and SOD as an oxidative enzyme scavenges reactive oxygen species [47]. Consistent with our results, gastric lipid peroxidation was increased in rats with aspirin- or alcohol-induced gastric mucosal injury [48-50]. The CPC and MIX groups significantly reduced gastric MDA levels, and the LBP group tended to decrease gastric MDA levels. A previous study found that LBP supplement $(100 \mathrm{mg} / \mathrm{kg} \mathrm{bw})$ for 24 weeks activated hepatic nuclear factor erythroid 2-related factor 2 (Nrf2) and reduced intracellular reactive oxygen species in C57BL/6 J mice with high fat diet-induced insulin resistance [51]. Therefore, LBP as an antioxidant [52] can attenuate oxidative stress via activating Nrf2 to inhibit oxidative stress-induced damage [53]. Whereas CPC as an antioxidant could also scavenge free radicals and inhibit microsomal and $\mathrm{CCl}_{4}$-induced lipid peroxidation in vitro and in vivo, respectively [54].

Aspirin or treatments did not change protein expression of gastric pro-inflammatory or anti-inflammatory markers in our study. A previous study showed that oral administration of aspirin in a single dose of $400 \mathrm{mg} / \mathrm{kg}$ bw significantly increased gastric TNF- $\alpha$, IL-6, and IL-12 levels, but significantly decreased gastric IL- 4 and IL-10 levels in rats with gastric mucosal damage caused by aspirin after $8 \mathrm{~h}$ of aspirin administration [10]. Similarly, oral administration of aspirin in a single dose of $200 \mathrm{mg} /$ kg bw significantly increased serum TNF- $\alpha$ and IL-1 $\beta$ levels in rats with gastric ulcer caused by aspirin after $4 \mathrm{~h}$ of aspirin administration [54]. The different results from our findings could be due to different duration of aspirin administration. In our study, oral administration of aspirin was chronic and continuous for 8 weeks, and the inflammatory response could not be increased after chronic use of aspirin because of the anti-inflammatory effect of aspirin. In the previous studies, gastric mucosa damage was caused by a single administration of aspirin and rats were sacrificed in a short time after aspirin administration $[10,55]$. Further studies are needed to evaluate whether mRNA expression of inflammatory markers could be regulated by aspirin or treatments at the transcriptional level.

The Firmicutes phylum was the most abundant phylum in gastric microbiota of rats with gastric ulcer caused by aspirin. The aspirin-treated groups tended to increase the relative abundance of the genus Streptococcus compared with the $\mathrm{N}$ group ( $3.7 \pm 3.5$ vs. $0.4 \pm 0.1 \%, p>0.05$ ), but there were no significant differences among five groups due to a large variation. Similarly, the Firmicutes phylum and the Streptococcus genus in the gastric biopsies from the patients with antral gastritis were more relative abundance compared with those in the normal biopsies [56]. Clostridium difficile in the Firmicutes phylum was related to the inflammatory response and bowel disease [57]. The patients with Clostridium difficile-associated diarrhea had significantly elevated mean platelet volume, neutrophil-lymphocyte ratio, and serum C-reactive protein levels compared with age- and sex-matched subjects with diarrhea but without Clostridium difficile infection [57]. However, whether the increase in the relative abundance of Firmicutes is causative for gastric ulcer or as a result of microbial changes due to gastric ulcer was remained to be further investigated. Consistent with our data, Firmicutes, Bacteroidetes, Proteobacteria, and Actinobacteria were predominant phyla in normal rat gastric microbiota [58]. At the genus level, Turicibacter, Lactobacillus, and Clostridium of the phylum Firmicutes were relatively abundant in normal rat gastric microbiota [58]. After aspirin administration, the genus Ruminococcaceae of the phylum Firmicutes was significantly enriched in rat stomach compared with the $\mathrm{N}$ or treated groups in our study. A previous study showed that family Ruminococcaceae, Prevotella species, Bacteroides species, and Barnesiella species were significantly discriminated in colonic microbiota of the adults using aspirin compared 
with those using no medication [59]. However, our study found that the relative abundance of the Firmicutes phylum in rat stomach was not significantly different among different groups except for the LBP group with lower relative abundance of the Firmicutes phylum as compared with the $\mathrm{N}$ group.

Our results revealed that supplementation of LBP and/ or CPC significantly increased the relative abundance of the genus Bifidobacterium in the Actinobacteria phylum. It is suspected that increased relative abundance of the genus Bifidobacterium in the LBP, CPC, and MIX groups could be associated with the changes in gastroprotective factors. However, no significant associations were observed between the relative abundance of the genus Bifidobacterium and the levels of gastroprotective factors in the stomach (Additional file 1: Table S1). Increased Bifidobacterium by both LBP and CPC could be a source of potential beneficial bacteria in the stomach for the host's health. The genus Bifidobacterium had protective effects on damaged gastric or intestinal mucosa in rats $[60,61]$ and humans [62]. Prophylactic administration of Bifidobacterium animalis VKL and VKB mixture for 14 days effectively protected against the development of ulcerative lesions in gastric mucosa caused by water immersion restraint stress in rats via preventing the degradation of mucus barrier [60]. The incidence of ileal ulcer developed by 5-bromo-2-(4-fluorophenyl)-3-(4methylsulfonylphenyl) thiophene (BFMeT, a NSAID) in rats was decreased by oral administration of Bifidobacterium adolescentis in drinking water through inhibiting the growth of aerobic bacteria in the ileum and decreasing the production of lipid peroxides in ileal mucosa [61]. Daily oral administration of Bifidobacterium breve capsules $\left(\geq 5 \times 10^{10}\right.$ colony-forming units) for 8 weeks significantly decreased the area under the curve for small intestinal damage (Lewis score) in adults with small intestinal lesions caused by 6-week oral intake of aspirin compared with those taking placebo without Bifidobacterium breve [62]. It is known that symbiotic bacteria in the intestine can break down non-digestible carbohydrates such as non-starch polysaccharides, resistant starch, oligosaccharides, and disaccharides, and further ferment these carbohydrates to release short-chain fatty acids [63]. These short-chain fatty acids produced by intestinal microbiota can be an energy source and decrease the luminal $\mathrm{pH}$ value, which is beneficial to the growth of probiotics and adverse to the growth of pathogens in the intestine [63]. An in vitro study found that LBP extract containing polysaccharides and polyphenols could be used as a source of food or nutraceuticals to help the growth of probiotics and protect the viability of Bifidobacterium and Lactobacillus in the simulated gastric and intestinal juices [64]. A previous study showed that LBP with major monosaccharides as glucuronic acid, galacturonic acid, glucose, galactose, and arabinose stimulated the production of short-chain fatty acids and increased the relative abundances of genera Bifidobacterium, Bacteroides, Phascolarctobacterium, Clostridium XIVb, Prevotella, and Collinsella in vitro after 24-h fermentation of LBP by healthy human fecal microbiota [19].

Supplementation of spirulina in vitro significantly promoted the growth of the lactic acid producing bacteria [65] because of the nitrogenous substance in spirulina [66]. Polyphenolic compounds in spirulina were also reported to regulate gut microbial communities [67]. In addition, oral ingestion of a dairy product containing Lactobacillus GG, Lactobacillus helveticus, and Lactobacillus acidophilus at a dose of $2.4 \times 10^{9} / \mathrm{strain} / \mathrm{d}$ for 5 days protected the integrity of the gastric mucosal barrier against the damage of indomethacin in healthy humans [68]. Our data indicated that CPC (a bioactive ingredient of spirulina) treatment significantly increased the class Actinobacteria, the order Bifidobacteriales, the family Bifidobacteriaceae, and the genus Bifidobacterium in the stomach. A recent study showed that daily oral pretreatment of phycocyanin $(50 \mathrm{mg} / \mathrm{kg} \mathrm{bw})$ for 30 days increased the proportion of beneficial bacteria such as the genera Lactobacillus, Bifidobacterium, and Roseburia and decreased that of harmful bacteria such as the genus Desulfovibrio in the phylum Proteobacteria in the cecum and feces of mice with radiation-induced acute gastrointestinal syndrome [20]. The pretreatment of phycocyanin improved ileal integrity via increasing tight junction proteins and decreased intestinal inflammatory signaling proteins in mice with radiation-induced acute gastrointestinal syndrome [20]. In addition, the abundance of the genera Roseburia, Enterorhabdus, Alistipes, Coriobacteriaceae, Lachnospiraceae, and Ruminococcaceae in the cecum and feces was negatively associated with histopathological indicators of intestinal injury, but positively correlated with the levels of tight junction proteins [20]. It is suggested that CPC could modulate gastric microbiota to increase beneficial bacteria, which might be associated with enhancing intestinal integrity. Therefore, LBP and/or CPC may protect against gastric ulcer caused by NSAIDs via improving the compositions of gastric microbiota.

The relative abundance of the phylum Bacteroidetes in the LBP and MIX groups tended to be slightly increased, but was not significantly different among five groups. The phyla Proteobacteria, Bacteroidetes, and Firmicutes were predominant in patients with Helicobacter pyloripositive gastric antrum ulcer or duodenal ulcer [69]. The phylum Proteobacteria was mostly abundant in gastric antrum of the patients with Helicobacter pylori-positive gastric antrum ulcer, and the phyla Bacteroidetes 
and Firmicutes were mostly abundant in the duodenum of patients with Helicobacter pylori-positive duodenal ulcers [69]. Our data showed that the phyla Firmicutes and Bacteroidetes were predominant in aspirin treated groups. The proportion of bacteria in the phylum was different between humans and rats and between gastric ulcer and duodenal ulcer. In addition, the relative abundance of bacteria could be affected by the etiology for the development of gastric ulcer such as Helicobacter pylori infection and chronic use of NSAIDs. Although certain Bacteroidetes species could be opportunistic pathogens, the phylum Bacteroidetes was considered to be the most stable component in the gastrointestinal microbiota of healthy adults and act as a symbiotic microorganism in the gastrointestinal tract to play a role in protein metabolism [70]. The limitation of this study was that we did not design the groups with the administration of LBP or CPC without aspirin, and we cannot determine the effects of LBP or CPC per se on gastric biochemical indicators. Therefore, it is required further studies to verify whether LBP or CPC could have the impact and molecular regulation on gastroprotective factors, antioxidant and inflammatory markers, and gastric microbiota.

\section{Conclusions}

In summary, after gastric ulcer caused by aspirin for 8 weeks in rats, LBP treatment for 9 weeks reduced gastric ulcer score, and CPC treatment for 9 weeks increased gastric total nitrite and nitrate levels and inhibited gastric lipid oxidation. The combination of LBP and CPC for 9 weeks elevated gastroprotective factors via increasing gastric COX-1, $\mathrm{PGE}_{2}$, and total nitrite and nitrate levels and enhanced antioxidative activities via inhibiting gastric lipid peroxidation. In addition, LBP and/or CPC improved gastric microbiota via increasing gastric Bifidobacterium. Therefore, CPC has gastroprotective effects on healing of gastric ulcer caused by aspirin, and combined LBP with CPC have better protection against gastric ulcer caused by aspirin in rats.

\section{Supplementary Information}

The online version contains supplementary material available at https://doi. org/10.1186/s12986-020-00538-9.

Additional file 1. Supplementary file: Fig. S1: Accumulated body weight gains of rats from week 1 to week 9, Fig. S2: Macroscopic and microscopic observations of rat stomach tissue, Fig. S3: Effects of Lycium barbarum polysaccharides (LBP) and/or C-phycocyanin (CPC) on inflammatory markers in stomach tissues, Fig. S4: The taxa distribution of rat gastric microbiota, Fig. S5: Beta diversity of gastric microbiota, Table S1: Correlation coefficients between the relative abundance of the genus Bifidobacterium and the levels of gastroprotective factors in the stomach.

\section{Abbreviations}

ANOVA: Analysis of variance; ASP: Aspirin; COX: Cyclooxygenase; CPC: C-phycocyanin; HPAEC-PAD: High pH anion exchange chromatography-pulsed amperometric detection; HRP: Horseradish peroxidase; ICAM-1: Intercellular adhesion molecule-1; IL: Interleukin; LBP: Lycium barbarum polysaccharides; LDA: Linear discriminant analysis; LEfSe: Linear discriminant analysis effect size; MDA: Malondialdehyde; NF-kB: Nuclear factor-kB; NGS: Next-generation sequencing; NO: Nitric oxide; NOx: Nitrite and nitrate; Nrf2: Nuclear factor erythroid 2-related factor 2; NSAIDs: Non-steroidal anti-inflammatory drugs; SOD: Superoxide dismutase; PCOA: Principal coordinate analysis; PCR: Polymerase chain reaction; PBS: Phosphate buffered saline; PGs: Prostaglandins; SEM: Standard error of means; TMB: 3,3',5,5'-Tetramethylbenzidine; TNF-a: Tumor necrosis factor-a; UI: Ulcer index.

\section{Acknowledgements}

The work was supported by the Ministry of Science and Technology, Taiwan, Republic of China (Grant Number MOST105-2320-B-038-036-MY3). The authors acknowledge Academia Sinica (Taipei, Taiwan) for the analysis of monosaccharide compositions of LBP and National Laboratory Animal Center for histopathological assessments of rat liver tissues.

\section{Authors' contributions}

SYH and JCJC were involved in the conceptualization and the design of this study. SYH conducted this study, and YZL, IHL, YCY, AAT, and AVS assisted biochemical and gastric microbiota analyses. All authors provided critical inputs to data analyses and the interpretation of the data. All authors read and approved the final manuscript.

\section{Funding}

The work was supported by the Ministry of Science and Technology, Taiwan, Republic of China (Grant Number MOST105-2320-B-038-036-MY3).

\section{Availability of data and materials}

The data are not publicly available. Nevertheless, the data are accessible from the authors upon reasonable request.

\section{Ethics approval and consent to participate}

All animal protocols were approved by the Institutional Animal Care and Use Committee at Taipei Medical University (LAC-2015-0214).

\section{Consent for publication}

Not applicable.

\section{Competing interests}

The authors declare that they have no competing interests.

\section{Author details}

${ }^{1}$ School of Nutrition and Health Sciences, College of Nutrition, Taipei Medical University, 250 Wu-Hsing Street, Taipei 110 11031, Taiwan. ${ }^{2}$ Research Center of Translational Medicine, Taipei Medical University, Taipei 110, Taiwan. ${ }^{3}$ Joint Biobank, Office of Human Research, Taipei Medical University, Taipei 110, Taiwan. ${ }^{4}$ Sechenov First Moscow State Medical University, Moscow, Russia. ${ }^{5}$ K.G. Razumovsky Moscow State University of Technologies and Management, Moscow, Russia. ${ }^{6}$ Master Program in Global Health and Development, Taipei Medical University, Taipei 110, Taiwan. ${ }^{7}$ Nutrition Research Center, Taipei Medical University Hospital, Taipei 110, Taiwan.

Received: 14 September 2020 Accepted: 26 December 2020 Published online: 06 January 2021

\section{References}

1. Chan FK, Leung WK. Peptic-ulcer disease. Lancet. 2002;360:933-41.

2. Lin KJ, García Rodríguez LA, Hernández-Díaz S. Systematic review of peptic ulcer disease incidence rates: do studies without validation provide reliable estimates? Pharmacoepidemiol Drug Saf. 2011;20:718-28.

3. Rosenstock SJ, Jørgensen T. Prevalence and incidence of peptic ulcer disease in a Danish County - a prospective cohort study. Gut. 1995:36:819-24. 
4. Kurata JH, Nogawa AN, Abbey DE, et al. A prospective study of risk for peptic ulcer disease in seventh-day adventists. Gastroenterology. 1992:102:902-9.

5. Wang FW, Tu MS, Mar GY, et al. Prevalence and risk factors of asymptomatic peptic ulcer disease in Taiwan. World J Gastroenterol. 2011;17:1199-203.

6. Amandeep K, Robin S, Ramica S, et al. Peptic ulcer: a review on etiology and pathogenesis. Int Res J Pharm. 2012;3:34-48.

7. Hsu PI, Tsai TJ. Epidemiology of upper gastrointestinal damage associated with low-dose aspirin. Curr Pharm Des. 2015;21:5049-55.

8. Tanaka A, Hase S, Miyazawa T, et al. Role of cyclooxygenase (COX)-1 and (COX)-2 inhibition in nonsteroidal anti-inflammatory drug-induced intestinal damage in rats: relation to various pathogenic events. J Pharmacol Exp Ther. 2002;303:1248-54

9. Cho $\mathrm{CH}$. Current roles of nitric oxide in gastrointestinal disorders. J Physiol Paris. 2001;95:253-6.

10. Raghavendran HRB, Srinivasan P, Rekha S. Immunomodulatory activity of fucoidan against aspirin-induced gastric mucosal damage in rats. Int Immunopharmacol. 2011;11:157-63.

11. Fan DD, Lin S, Song YP, et al. Astragaloside IV protects rat gastric mucosa against aspirin-induced damage. Int Immunopharmacol. 2016;41:47-55

12. Wu P-S, Wu S-J, Tsai Y-H, et al. Hot water extracted Lycium barbarum and Rehmannia glutinosa inhibit liver inflammation and fibrosis in rats. Am J Chin Med. 2011;39:1173-91

13. Chai S, Lee S, Ng G. Gou Qi Zi and its chemical composition. Chin Pharmacol Bull. 1986;11:41-3.

14. Gan L, Zhang SH, Yang XL, et al. Immunomodulation and antitumor activity by a polysaccharide-protein complex from Lycium barbarum. Int Immunopharmacol. 2004;4:563-9.

15. Jin M, Huang Q, Zhao K, et al. Biological activities and potential health benefit effects of polysaccharides isolated from Lycium barbarum L. Int J Biol Macromol. 2013;54:16-23.

16. Kuddus $M$, Singh $P$, Thomas $G$, et al. Recent developments in production and biotechnological applications of C-phycocyanin. Biomed Res Int. 2013;2013:742859.

17. Madhyastha HK, Radha KS, Nakajima Y, et al. UPA dependent and independent mechanisms of wound healing by C-phycocyanin. J Cell Mol Med. 2008;12:2691-703.

18. Zheng J, Inoguchi T, Sasaki S, et al. Phycocyanin and phycocyanobilin from Spirulina platensis protect against diabetic nephropathy by inhibiting oxidative stress. Am J Physiol Regul Integr Comp Physiol. 2013:304:R110-20.

19. Ding $Y$, Yan $Y$, Peng $Y$, et al. In vitro digestion under simulated saliva, gastric and small intestinal conditions and fermentation by human gut microbiota of polysaccharides from the fruits of Lycium barbarum. Int J Biol Macromol. 2019;125:751-60.

20. Lu L, LiW, Sun C, et al. Phycocyanin ameliorates radiation-induced acute intestinal toxicity by regulating the effect of the gut microbiota on the TLR4/Myd88/NF-kB pathway. J Parenter Enteral Nutr. 2019. https://doi. org/10.1002/jpen.1744.

21. Cui B, Liu S, Lin X, et al. Effects of Lycium barbarum aqueous and ethano extracts on high-fat-diet induced oxidative stress in rat liver tissue. Molecules. 2011;16:9116-28.

22. Shih CM, Cheng SN, Wong CS, et al. Antiinflammatory and antihyperalgesic activity of C-phycocyanin. Anesth Analg. 2009;108:1303-10.

23. Sun YX, Zhang J, Yu GC, et al. Experimental study on the therapeutic effect of C-phycocyanin against pulmonary fibrosis induced by paraquat in rats. Zhonghua Lao Dong Wei Sheng Zhi Ye Bing Za Zhi. 2012;30:650-5.

24. Chiang YY, Chao JC-J. Olive oil combined with Lycium barbarum polysaccharides attenuates liver apoptosis and inflammation induced by carbon tetrachloride in rats. J Funct Foods. 2018;48:329-36.

25. Ganguly A, Bhatnagar O. Effect of bilateral adrenalectomy on production of restraint ulcers in the stomach of albino rats. Can J Physiol Pharmacol. 1973;51:748-50.

26. Takeuchi K, Ohishi M, Endo K, et al. Hydroxyproline, a serum biomarker candidate for gastric ulcer in rats: a comparison study of metabolic analysis of gastric ulcer models induced by ethanol, stress, and aspirin. Biomark Insights. 2014;9:61-6.

27. Sadegh M, Sakhaie MH. Carvacrol mitigates proconvulsive effects of lipopolysaccharide, possibly through the hippocampal cyclooxygenase-2 inhibition. Metab Brain Dis. 2018:33:2045-50.
28. HuTY, Ju JM, Mo LH, et al. Anti-inflammation action of xanthones from Swertia chirayita by regulating COX-2/NF-KB/MAPKs/Akt signaling pathways in RAW 264.7 macrophage cells. Phytomedicine. 2019;55:214-21.

29. Sun J, Zhang $X$, Broderick $M$, et al. Measurement of nitric oxide production in biological systems by using Griess reaction assay. Sensors. 2003;3:276-84.

30. Bradford MM. A rapid and sensitive method for the quantitation of microgram quantities of protein utilizing the principle of protein-dye binding Anal Biochem. 1976:72:248-54.

31. Nebot C, Moutet M, Huet P, et al. Spectrophotometric assay of superoxide dismutase activity based on the activated autoxidation of a tetracyclic catechol. Anal Biochem. 1993;214:442-51.

32. Yagi K. Simple assay for the level of total lipid peroxides in serum or plasma. Methods Mol Biol. 1998:108:101-6.

33. Zaki MH, Vogel P, Malireddi RK, et al. The NOD-like receptor NLRP12 attenuates colon inflammation and tumorigenesis. Cancer Cell. 2011;20:649-60.

34. Zhong X, Li X, Liu F, et al. Omentin inhibits TNF-a-induced expression of adhesion molecules in endothelial cells via ERK/NF-KB pathway. Chemosphere. 2019;223:675-85

35. Ten Hove T, Corbaz A, Amitai H, et al. Blockade of endogenous IL-18 ameliorates TNBS-induced colitis by decreasing local TNF-a production in mice. Gastroenterology. 2001;121:1372-9.

36. Quast C, Pruesse E, Yilmaz P, et al. The SILVA ribosomal RNA gene database project: improved data processing and web-based tools. Nucleic Acids Res. 2013;41(Database issue):D590-6.

37. Schliep KP. Phangorn: phylogenetic analysis in R. Bioinformatics. 2011;27:592-3

38. McMurdie PJ, Holmes S. Phyloseq: an R package for reproducible interactive analysis and graphics of microbiome census data. PLOS ONE. 2013:8:e61217.

39. Chen J, Bittinger $\mathrm{K}$, Charlson ES, et al. Associating microbiome composition with environmental covariates using generalized UniFrac distances. Bioinformatics. 2012;28:2106-13.

40. Asnicar F, Weingart G, Tickle TL, et al. Compact graphical representation of phylogenetic data and metadata with GraPhIAn. PeerJ. 2015;3:e1029.

41. Segata N, Izard J, Waldron L, et al. Metagenomic biomarker discovery and explanation. Genome Biol. 2011;12:R60.

42. Takeuchi K, Ohishi M, Endo K, et al. Metabolic profiling to identify potential serum biomarkers for gastric ulceration induced by nonsteroid anti-inflammatory drugs. J Proteome Res. 2013;12:1399-407.

43. Ramakrishnan K, Salinas RC. Peptic ulcer disease. Am Fam Phys. 2007;76:1005-12

44. Li C, Yu Y, Li W, et al. Phycocyanin attenuates pulmonary fibrosis via the TLR2-MyD88-NF-kB signaling pathway. Sci Rep. 2017;7:5843.

45. Wallace JL. Prostaglandins, NSAIDs, and gastric mucosal protection: why doesn't the stomach digest itself? Physiol Rev. 2008;88:1547-65.

46. Dejban P, Eslami F, Rahimi N, et al. Involvement of nitric oxide pathway in the anti-inflammatory effect of modafinil on indomethacin-, stress-, and ethanol-induced gastric mucosal injury in rat. Eur J Pharmacol. 2020:887:173579.

47. Bhattacharyya A, Chattopadhyay R, Mitra S. Oxidative stress: an essential factor in the pathogenesis of gastrointestinal mucosal diseases. Physiol Rev. 2014;94:329-54.

48. Ajaikumar KB, Asheef $M, B a b u B H$, et al. The inhibition of gastric mucosal injury by Punica granatum L. (pomegranate) methanolic extract. J Ethnopharmacol. 2005;96:171-6.

49. D'Argenio G, Mazzone G, Tuccillo C, et al. Apple polyphenol extracts prevent aspirin-induced damage to the rat gastric mucosa. Br J Nutr. 2008:100:1228-36

50. Cuevas VM, Calzado YR, Guerra YP, et al. Effects of grape seed extract, vitamin C, and vitamin E on ethanol-and aspirin-induced ulcers. Adv Pharmacol Sci. 2011;2011:740687.

51. Yang $Y$, Wang L, Li Y, et al. Dietary Lycium barbarum polysaccharide induces nrf2/ARE pathway and ameliorates insulin resistance induced by high-fat via activation of PI3K/AKT signaling. Oxid Med Cell Longev. 2014;2014:145641.

52. Skenderidis P, Kerasioti E, Karkanta E, et al. Assessment of the antioxidant and antimutagenic activity of extracts from goji berry of Greek cultivation. Toxicol Rep. 2018;5:251-7. 
53. Cheng J, Zhou ZW, Sheng HP, et al. An evidence-based update on the pharmacological activities and possible molecular targets of Lycium barbarum polysaccharides. Drug Des Devel Ther. 2015;9:33-78.

54. Romay Ch, González R, Ledón N, et al. (2003) C-phycocyanin: a biliprotein with antioxidant, anti-inflammatory and neuroprotective effects. Curr Protein Pept Sci. 2003;4:207-16.

55. Wang $Z$, Hasegawa J, Wang $X$, et al. Protective effects of ginger against aspirin-induced gastric ulcers in rats. Yonago Acta Med. 2011;54:11-9.

56. Li X-X, Wong GL-H, To K-F, et al. Bacterial microbiota profiling in gastritis without Helicobacter pylori infection or non-steroidal anti-inflammatory drug use. PLoS ONE. 2009;4:e7985.

57. Nseir W, Khamisy-Farah R, Amara A, et al. The prognostic value of inflammatory markers in Clostridium difficile-associated diarrhea. Isr Med Assoc J. 2019;21:658-61.

58. Li D, Chen H, Mao B, et al. Microbial biogeography and core microbiota of the rat digestive tract. Sci Rep. 2017:8:45840.

59. Rogers MAM, Aronoff DM. The influence of non-steroidal antiinflammatory drugs on the gut microbiome. Clin Microbiol Infect. 2016;22(178):e1-9.

60. Spivak M, Lazarenko LM, Falalieieva TM, et al. Prophylactic effect of probiotic strains Bifidobacterium animalis VKL and VKB on stress-induced lesions in the gastric mucosa of rats. Fiziol Zh. 2013;59:23-30.

61. Kinouchi T, Kataoka K, Bing SR, et al. Culture supernatants of Lactobacillus acidophilus and Bifidobacterium adolescentis repress ileal ulcer formation in rats treated with a nonsteroidal antiinflammatory drug by suppressing unbalanced growth of aerobic bacteria and lipid peroxidation. Microbiol Immunol. 1998;42:347-55.

62. Mortensen B, Murphy C, O'Grady J, et al. Bifidobacterium breve Bif195 protects against small-intestinal damage caused by acetylsalicylic acid in healthy volunteers. Gastroenterology. 2019;157(637-46):e4.
63. Blaut M. Relationship of prebiotics and food to intestinal microflora. Eur J Nutr. 2002;41(Suppl 1):i11-6.

64. Skenderidis P, Mitsagga C, Lampakis D, et al. The Effect of encapsulated powder of goji berry (Lycium barbarum) on growth and survival of probiotic bacteria. Microorganisms. 2019;8:57. https://doi.org/10.3390/micro organisms8010057.

65. Parada JL, de Caire GZ, de Mule MCZ, et al. Lactic acid bacteria growth promoters from Spirulina platensis. Int J Food Microbiol. 1998;45:225-8.

66. Beheshtipour H, Mortazavian AM, Mohammadi R, et al. Supplementation of Spirulina platensis and Chlorella vulgaris algae into probiotic fermented milks. Compr Rev Food Sci Food Saf. 2013;12:144-54.

67. Peluso I, Romanelli L, Palmery M. Interactions between prebiotics, probiotics, polyunsaturated fatty acids and polyphenols: diet or supplementation for metabolic syndrome prevention? Int J Food Sci Nutr. 2014;65:259-67.

68. Gotteland M, Cruchet S, Verbeke S. Effect of Lactobacillus ingestion on the gastrointestinal mucosal barrier alterations induced by indometacin in humans. Aliment Pharmacol Ther. 2001;15:11-7.

69. Chen X, Xia C, Li Q, et al. Comparisons between bacterial communities in mucosa in patients with gastric antrum ulcer and a duodenal ulcer. Front Cell Infect Microbiol. 2018;8:126.

70. Rajilić-Stojanović M, de Vos WM. The first 1000 cultured species of the human gastrointestinal microbiota. FEMS Microbiol Rev. 2014;38:996-1047.

\section{Publisher's Note}

Springer Nature remains neutral with regard to jurisdictional claims in published maps and institutional affiliations.
Ready to submit your research? Choose BMC and benefit from:

- fast, convenient online submission

- thorough peer review by experienced researchers in your field

- rapid publication on acceptance

- support for research data, including large and complex data types

- gold Open Access which fosters wider collaboration and increased citations

- maximum visibility for your research: over $100 \mathrm{M}$ website views per year

At BMC, research is always in progress.

Learn more biomedcentral.com/submissions 